\title{
Mixed-measurement of Zero-sequence Parameters of Parallel Coupled Transmission lines Based on Variable-frequency Electrical Measurement
}

\author{
Shengsuo Niu ${ }^{a}$, Jia Zhang ${ }^{b}$ and Zhirui Liang ${ }^{c}$ \\ North China Electric Power University, Baoding, Hebei, 071003, China \\ aniushengsuo@163.com, ${ }^{b} x j r 900 @$ sina.com, cliangzr0312@163.com
}

Keywords: zero-sequence parameters, mixed-measurement, variable-frequency measurement, simulation.

\begin{abstract}
Based on the demand of real projects that new overhead wires are been erected on the running wires with power flow, if zero-sequence parameters of the wires are needed, zero-sequence signals of different frequencies around the basic $50 \mathrm{~Hz}$ can be added to the newly erected ones to generate zero-sequence signals, avoiding the short-term power down of the running wires. This paper analyses the structure of multi-coupled lines with different voltage levels, builds "double-П-shaped" zero sequence lumped-parameter circuit model, proposes a new algorithm based on variable-frequency electrical measurement to work out the zero-sequence parameters, including self-impedance, admittance and mutual parameters of the coupled lines. Simulation experiments for double circuit and triple circuit of different voltage levels are carried out in this paper. The simulation results reveal that the circuit models and zero-sequence parameter measurement algorithms are applicable to the zero-sequence parameters measurement of power system.
\end{abstract}

\section{Introduction}

As the carrier of power transmission, transmission lines are a great part of power system. The accuracy of line parameters are essential for power flow calculation, relay setting calculation, fault analysis, fault allocation and selection of power system operation mode [1, 2, 3]. Zero-sequence parameters are the basis of zero sequence line protection calculation, and it is demanded to be measured through real tests $[2,4,5]$.

With the development of power construction, the capacity of power system is getting bigger, voltage level higher, transmission line corridor narrower, which increases the zero sequence mutual impedance and admittance [6, 7]. The traditional methods of calculating line parameters are formula method, off-line measurement method and on-line measurement method [3]. The uncertain parameters in the formula involves the earth resistivity and the equivalent depth which will change with the geographical conditions, thus the formula calculation results always have great error with actual values. Off-line measurement requires that all the routes are off power. This method has high accuracy but limited in practice [5]. Online measurement is most widely used currently [1, 2, 7-9].

The mixed-measurement of zero-sequence parameters of parallel coupled transmission lines based on variable-frequency electrical measurement this paper proposed is used specifically for line parameter measurement of coupled lines with newly built lines [10]. Zero-sequence signals of different frequencies can be superimposed and added to the newly built lines. All data can be collected in one time. By adopting filtering software and hardware, the amplitude and phase angle of each signal can be accurately captured, and can be used to work out the parameters. This approach guarantees the accuracy of the results, while reducing the number of measurements, and does not affect the normal operation of the line of work. 


\section{A zero sequence circuit model for parallel transmission lines}

\subsection{The zero sequence circuit models.}

According to the current level of development of the grid, the case of coupled lines includes two main lines and three lines. Take two lines as example. The two parallel coupled transmission lines have different parameters and are applied to different voltage levels. Assuming that lines have gone through three-segment transposition or nine-segment transposition, so as that self-impedance are the same for each conductor from the same circuit and mutual impedance from a certain conductor of line 1 to another in line 2 is also the same. Based on circuit analysis theory, we build the model of two parallel lines, seen as fig.1, showing the relations of voltage drops and current drops with impedance and admittance. In the model, we use the traditional "double-П-shaped" circuit model, three coupled lines has the similar model. For the parameters, we have self-impedance and admittance to ground, mutual impedance (including mutual impedances and admittance between phase currents and between adjacent lines). The work of conductivity is negligible because of the little effect it can cause. Considering the impact between lines, mutual impedance between adjacent lines does more effects. The key point is zero sequence self-impedance and admittance of the new built line and the mutual impedance and admittance between adjacent lines.

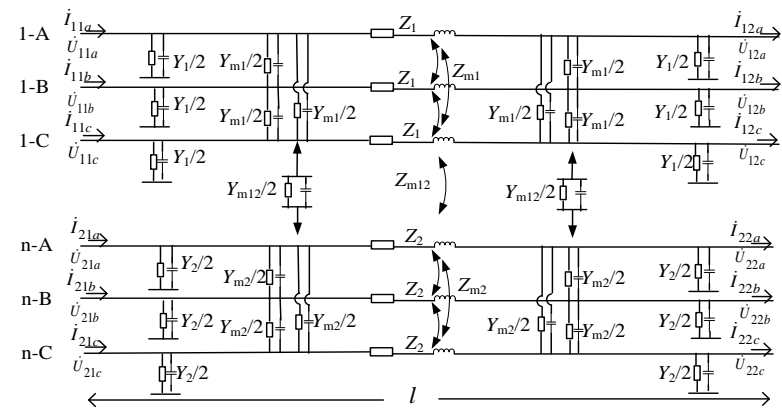

Fig.1 two parallel transmission line model

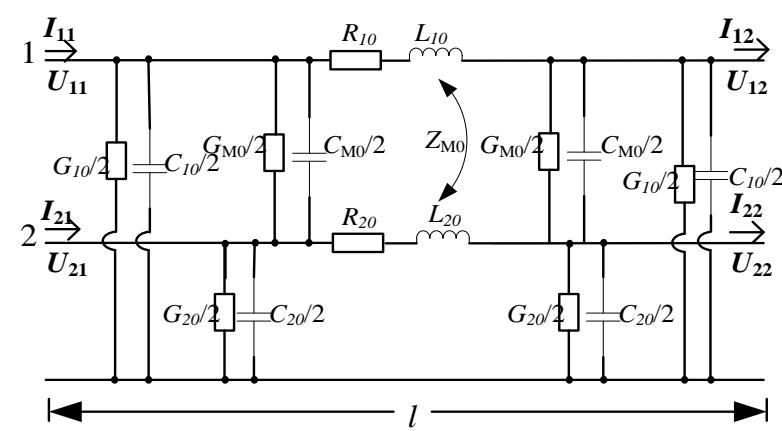

Fig.2 "Double-П-shaped" zero-sequence lumped-parameter circuit model

\subsection{Translation from ABC domain into 012 domain.}

Based on fig.1, we can make equations based on voltage drops and current drops from both lines, seen as below.

$$
\left\{\begin{array}{l}
\mathrm{I}_{1 \mathrm{abc}}-\mathrm{I}_{2 \mathrm{abc}}=\frac{1}{2} \mathrm{Y}_{\mathrm{abc}}\left(\mathrm{U}_{1 \mathrm{abc}}+\mathrm{U}_{2 \mathrm{abc}}\right) \\
\mathrm{U}_{1 \mathrm{abc}}-\mathrm{U}_{2 \mathrm{abc}}=\mathrm{Z}_{\mathrm{abc}}\left(\mathrm{I}_{1 \mathrm{abc}}-\frac{1}{2} \mathrm{Y}_{\mathrm{abc}} \mathrm{I}_{2 \mathrm{abc}}\right)
\end{array}\right.
$$

We wish to covert (1) to the 012 domain equations. Recall the matrices that are used in the conversion from $\mathrm{ABC}$ to 012 domain, and vice versa are 


$$
\mathrm{A}=\frac{1}{3}\left[\begin{array}{ccc}
1 & \mathrm{a} & \mathrm{a}^{2} \\
1 & \mathrm{a}^{2} & \mathrm{a} \\
1 & 1 & 1
\end{array}\right] \text { and } \mathrm{A}^{-1}=\left[\begin{array}{ccc}
1 & 1 & 1 \\
\mathrm{a}^{2} & \mathrm{a} & 1 \\
\mathrm{a} & \mathrm{a}^{2} & 1
\end{array}\right]
$$

Where $\mathrm{a}=-\frac{1}{2}+j \frac{\sqrt{3}}{2}$

The conversion of $A B C$ domain and 012 domain is, take $U$ for instance, $U_{120}=A * U_{a b c}$, in turn, $\mathrm{U}_{\mathrm{abc}}=\mathrm{A}^{-1} * \mathrm{U}_{120}$. In this case, we use $\mathrm{A}^{-1} * \mathrm{U}_{120}$ to substitute $\mathrm{U}_{\mathrm{abc}}$, and we get

$$
\left\{\begin{array}{l}
\mathrm{A}^{-1} \mathrm{I}_{1120}-\mathrm{A}^{-1} \mathrm{I}_{2120}=\frac{1}{2} \mathrm{Y}_{\mathrm{abc}}\left(\mathrm{A}^{-1} \mathrm{U}_{1120}+\mathrm{A}^{-1} \mathrm{U}_{2120}\right) \\
\mathrm{A}^{-1} \mathrm{U}_{1120}-\mathrm{A}^{-1} \mathrm{U}_{2120}=\mathrm{Z}_{\mathrm{abc}}\left(\mathrm{A}^{-1} \mathrm{I}_{1120}-\frac{1}{2} \mathrm{Y}_{\mathrm{abc}} \mathrm{A}^{-1} \mathrm{I}_{2120}\right)
\end{array}\right.
$$

By simplifying (3), we get the final equation

$$
\left\{\begin{array}{l}
\mathrm{I}_{1120}-\mathrm{I}_{2120}=\frac{1}{2} \mathrm{Y}_{120}\left(\mathrm{U}_{1120}+\mathrm{U}_{2120}\right) \\
\mathrm{U}_{1120}-\mathrm{U}_{2120}=\mathrm{Z}_{120}\left(\mathrm{I}_{1120}-\frac{1}{2} \mathrm{Y}_{120} \mathrm{I}_{2120}\right)
\end{array}\right.
$$

Where $\mathrm{Z}_{120}=\mathrm{A} * \mathrm{Z}_{\mathrm{abc}} * \mathrm{~A}^{-1}, \mathrm{Y}_{120}=\mathrm{A} * \mathrm{Y}_{\mathrm{abc}} * \mathrm{~A}^{-1}$

Now we have converted ABC domain into 012 domain, we can calculate zero sequence parameters by using only zero sequence voltage and current.

\section{The principle of mixed measurement}

The measurement is proposed based on real projects. When the grid expands regional transmission capacity, new lines will be erectly built on existed ones. In calculating the power frequency zero-sequence parameters in such cases, one mixed signal of different frequencies is added to three phases of the newly built lines, and zero-sequence signal is induced on the running lines. All zero-sequence signals of both ends of the lines are been measured with PMU technology and zero-sequence parameters are been worked out with line topology. The advantage of this mixed measurement is that zero-sequence signal is been applied on the lines and adopted just for one time without powering off.

The unknown zero-sequence parameters of multi-coupled transmission lines are self and mutual impedance and admittance. Given the number of unknown parameters, we consider the frequency characteristics of inductance and calculate resistance and inductance separately. In this way we can get enough equations to work out all the parameters. When signals of different frequencies are added to the system, the impedance will change with the frequency, thus these equations of different frequencies are nonrelated and the equation set is solvable.

Take the parameter measurement of double coupled transmission lines as instance to have detailed instruction of variable-frequency measurement. The unknown parameters are zero-sequence self-impedance, self- admittance, and mutual-impedance and mutual admittance. The improved formula are as (5).

$$
\left\{\begin{array}{l}
I_{11}-I_{12}=\frac{1}{2} j 2 \pi f C_{10}\left(U_{11}+U_{12}\right)+\frac{1}{2} j 2 \pi f C_{m 0}\left(U_{11}-U_{21}+U_{12}-U_{22}\right) \\
I_{21}-I_{22}=\frac{1}{2} j 2 \pi f C_{20}\left(U_{21}+U_{22}\right)-\frac{1}{2} j 2 \pi f C_{m 0}\left(U_{11}-U_{21}+U_{12}-U_{22}\right) \\
U_{11}-U_{12}=\left(R_{10}+j 2 \pi f L_{10}\right)\left(I_{11}-\frac{1}{2} j 2 \pi f C_{10} U_{11}-\frac{1}{2} j 2 \pi f C_{m 0}\left(U_{11}-U_{21}\right)\right)+ \\
\left(R_{m 0}+j 2 \pi f L_{m 0}\right)\left(I_{21}-\frac{1}{2} j 2 \pi f C_{20} U_{21}+\frac{1}{2} j 2 \pi f C_{m 0}\left(U_{11}-U_{21}\right)\right) \\
U_{21}-U_{22}=\left(R_{20}+j 2 \pi f L_{20}\right)\left(I_{21}-\frac{1}{2} j 2 \pi f C_{20} U_{21}+\frac{1}{2} j 2 \pi f C_{m 0}\left(U_{11}-U_{21}\right)\right)+ \\
\left(R_{m 0}+j 2 \pi f L_{m 0}\right)\left(I_{11}-\frac{1}{2} j 2 \pi f C_{10} U_{11}-\frac{1}{2} j 2 \pi f C_{m 0}\left(U_{11}-U_{21}\right)\right)
\end{array}\right.
$$


In the formula, $R_{10}, R_{20}, R_{m 0}, L_{10}, L_{20}, L_{m 0}, C_{10}, C_{20}, C_{m 0}$ are parameters to be solved. $U_{11}, U_{12}$, $U_{21}, U_{22}, I_{11}, I_{12}, I_{21}, I_{22}$ are zero-sequence voltage value and current value of both ends of the line. The measurement under one frequency can get 4 equations not enough to work out 9 parameters. We need no less than 3 measurements of different frequencies.

Because the zero-sequence current passes through the earth, considering the skin effect of the wire and the earth, the zero-sequence parameters change with the frequency. Referring to some relevant articles that zero-sequence parameters change little within $0.005 \%$ with frequency from $40 \mathrm{~Hz}$ to 60 $\mathrm{Hz}$. Based on all the information, we adopt the data under $45 \mathrm{~Hz}, 50 \mathrm{~Hz}$ and $55 \mathrm{~Hz}$.

Set the signal as the sum of three signals of different frequencies and add to newly built wires, capture voltage and current wave of both ends via PMU, analyze the signal through Fourier transform and get the amplitude and angle of the signal of different frequency respectively.

4 equations can be written under every frequency and we have 9 equations in total. To work out this set we use least square method. Use the first two equations to rewrite capacitance matrix as equations (6) to (8).

$$
\begin{aligned}
& A_{1} \cdot C=\Delta I \\
& {\left[\begin{array}{lll}
u_{11}^{(1)}+u_{12}^{(1)} & u_{11}^{(1)}+u_{12}^{(1)}-u_{21}^{(1)}-u_{22}^{(1)} & 0 \\
0 & -\left(u_{11}^{(1)}+u_{12}^{(1)}-u_{21}^{(1)}-u_{22}^{(1)}\right) & u_{21}^{(1)}+u_{22}^{(1)} \\
u_{11}^{(2)}+u_{12}^{(2)} & u_{11}^{(2)}+u_{12}^{(2)}-u_{21}^{(2)}-u_{22}^{(2)} & 0 \\
0 & -\left(u_{11}^{(2)}+u_{12}^{(2)}-u_{21}^{(2)}-u_{22}^{(2)}\right) & u_{21}^{(2)}+u_{22}^{(2)} \\
u_{11}^{(3)}+u_{12}^{(3)} & u_{11}^{(3)}+u_{12}^{(3)}-u_{21}^{(3)}-u_{22}^{(3)} & 0 \\
0 & -\left(u_{11}^{(3)}+u_{12}^{(3)}-u_{21}^{(3)}-u_{22}^{(3)}\right) & u_{21}^{(3)}+u_{22}^{(3)}
\end{array}\right]\left[\begin{array}{l}
C_{10} \\
C_{m 0} \\
C_{20}
\end{array}\right]=2 \times\left[\begin{array}{l}
\left(i_{11}^{(1)}-i_{12}^{(1)}\right) / 2 \pi f^{(1)} \\
\left(i_{21}^{(1)}-i_{22}^{(1)}\right) / 2 \pi f^{(1)} \\
\left(\begin{array}{l}
(1) \\
(1)
\end{array} i_{12}^{(2)}\right) / 2 \pi f^{(2)} \\
\left(i_{21}^{(2)}-i_{22}^{(2)}\right) / 2 \pi f^{(2)} \\
\left.i_{11}^{(3)}-i_{12}^{(3)}\right) / 2 \pi f^{(3)} \\
\left(i_{21}^{(3)}-i_{22}^{(3)}\right) / 2 \pi f^{(3)}
\end{array}\right]} \\
& C=\left(A_{1}^{T} A_{1}\right)^{-1} A_{1}^{T} \Delta I
\end{aligned}
$$

The superscript in the matrix stands for the measurement of different frequencies. Transform the capacitance value into admittance and put it in the matrix formed with the last two equations in formula (5) and work out resistance and inductance value.

$A_{2} \cdot R L=\Delta U$

$$
R L=\left(A_{2}^{T} A_{2}\right)^{-1} A_{2}^{T} \Delta U
$$

In equation (10), $R L=\left[\begin{array}{llllll}R_{10} & L_{10} & R_{m 0} & L_{m 0} & R_{20} & L_{20}\end{array}\right]^{T}$

\section{The Example}

To verify mixed-measurement of zero-sequence parameters of parallel coupled transmission lines based on variable-frequency electrical measurement, we build a three parallel coupled lines system in MATLAB to have a digital simulation. The system is based on real projects. The voltage levels of three parallel lines are $2200 \mathrm{kV}, 220 \mathrm{kV}$ and $110 \mathrm{kV}$ respectively. Each line is equipped with matching transformers and loads. For the parallel line block we use distributed parameter lines and the parallel distance is $40 \mathrm{~km}$.

To provide zero sequence signal, one solution is to add external zero sequence voltage source directly on the off power lines, the other is to create single phase break line fault on the running lines. We adopt the least square method to calculate the equations.

The zero-sequence self-impedance and admittance and mutual impedance and admittance are the main targets in the calculation. The setting values, measuring values and errors of zero sequence parameters are shown in table 1 below. 
Table.1 Settings, results and error of simulation based on short-line zero-sequence distributed-parameter measurement algorithm

\begin{tabular}{ccccc}
\hline \multicolumn{2}{c}{$\begin{array}{c}\text { parameters } \\
(/ \mathrm{km})\end{array}$} & setting values & Measuring values & Error \% \\
\hline 220kV & $\mathrm{R}_{10}(\Omega)$ & 0.19379 & 0.18051 & 6.85 \\
Self-parameters & $\mathrm{L}_{10}\left(10^{-3} \mathrm{H}\right)$ & 2.40188 & 2.41235 & 0.43 \\
& $\mathrm{C}_{10}\left(10^{-8} \mathrm{~F}\right)$ & 0.72169 & 0.72002 & 0.23 \\
220kV and 220kV & $\mathrm{R}_{\mathrm{m} 12}(\Omega)$ & 0.15485 & 0.14961 & 3.38 \\
Mutual-parameters & $\mathrm{L}_{\mathrm{m} 12}\left(10^{-3} \mathrm{H}\right)$ & 1.18804 & 1.17453 & 1.13 \\
& $\mathrm{C}_{\mathrm{m} 12}\left(10^{-8} \mathrm{~F}\right)$ & 0.23069 & 0.23151 & 0.35 \\
220kV and 110kV & $\mathrm{R}_{\mathrm{m} 13}(\Omega)$ & 0.14199 & 0.14932 & 5.16 \\
Mutual-parameters & $\mathrm{L}_{\mathrm{m} 13}\left(10^{-3} \mathrm{H}\right)$ & 2.41772 & 2.40531 & 0.51 \\
& $\mathrm{C}_{\mathrm{m} 13}\left(10^{-8} \mathrm{~F}\right)$ & 0.05902 & 0.05948 & 0.77 \\
220kV & $\mathrm{R}_{20}(\Omega)$ & 0.20596 & 0.21035 & 2.13 \\
Self-parameters & $\mathrm{L}_{20}\left(10^{-3} \mathrm{H}\right)$ & 4.02693 & 4.01974 & 0.17 \\
& $\mathrm{C}_{20}\left(10^{-8} \mathrm{~F}\right)$ & 0.35941 & 0.35599 & 0.95 \\
220kV and 110kV & $\mathrm{R}_{\mathrm{m} 23}(\Omega)$ & 0.14108 & 0.14759 & 4.61 \\
Mutual-parameters & $\mathrm{L}_{\mathrm{m} 23}\left(10^{-3} \mathrm{H}\right)$ & 2.66661 & 2.66717 & 0.02 \\
& $\mathrm{C}_{\mathrm{m} 23}\left(10^{-8} \mathrm{~F}\right)$ & 0.18881 & 0.19012 & 0.69 \\
110kV & $\mathrm{R}_{30}(\Omega)$ & 0.30039 & 0.31259 & 4.0 \\
Self-parameters & $\mathrm{L}_{30}\left(10^{-3} \mathrm{H}\right)$ & 4.48693 & 4.4967 & 0.21 \\
& $\mathrm{C}_{30}\left(10^{-8} \mathrm{~F}\right)$ & 0.22803 & 0.23087 & 1.24 \\
\hline
\end{tabular}

\section{Conclusion}

From table 1 we can see, the measuring values of the zero-sequence parameters are very close to the setting values, the errors of resistance are a little larger that is because the weight the resistance takes is much less than inductance.

The mixed-measurement of zero-sequence parameters of parallel coupled transmission lines based on variable-frequency electrical measurement is proved to be feasible.

\section{References}

[1] LIANG Zhirui, GONG Ruibang, NIU Shengsuo, et al. Study on measuring system for power frequency parameters of power transmission line. Electric Power Automation Equipment. Vol. 33 (2013) No. 7, p. 70-74.

[2] GONG Ruibang. Study on Power Frequency Online Measurement of Transmission Line. Master, North China Electric Power University, China, 2013.

[3] QIN Wenlong. Research of parameter measurement of transmission lines. Master, Southwest Jiaotong University, China, 2011.

[4] HU Zhijian, CHENG Xiaomin. Measurement methods of zero-sequence impedance parameters for transmission lines. Journal of Electric Power Science and Technology. Vol. 25 (2010) No. 3, p. 25-31.

[5] HUANG Chao. Research on transmission line power frequency parameters. Master, Nanjing University of Science and Technology, China, 2010.

[6] LI Zhusen, CHEN Xiaoyan, WU Yanjiao, et al. Live line measurement of several parallel transmission lines. Automation of Electric Power Systems. Vol. 33 (2009) No. 22, p. 84-88.

[7] HU Zhijian, ZHANG Chifei, CHEN Yunping, et al. Measurement of mutual inductance parameters of long-distance high-voltage cable lines. Vol. 28 (2002) No. 5, p. 17-18. 
[8] WANG Chunna, LIANG Zhirui, XUE Zhiying, et al. Live line measurement of zero sequence parameters for transmission lines with mutual inductance. Electric Power Automation Equipment. Vol. 28 (2008) No. 4, p.87-90.

[9] LIANG Zhirui, WU Changqing, SU Haifeng, et al. A parameter measuring method of coupling transmission lines. Vol. 40 (2012) No. 8, p.126-130.

[10]HU Ning, HU Zhijian, ZHENG Gang, et al. Live line parameter measurement of transmission lines with mutual inductance based on GPS technology and integral equations. Power System Technology. Vol. 29 (2005) No. 16, p. 76-80.

[11]WEI Heng, ZHOU Di, WANG Yi, et al. Online parameter measurement of double-circuit transmission lines based on fault recorder. Power System Protection and Control. Vol. 39 (2011) No. 23, p.138-142.

[12]CHEN Guoqing, SUN Caixin, ZHANG Zhijin, et al. Study on the calculation model of lightning protection performance of shielding failure for $500 \mathrm{kV}$ double circuit transimission line. Proceeding of the SDEE. Vol. 23 (2003) No. 5, p.108-111.

[13]HU Zhijian, CHEN Yunping, et al. New method of live line measuring the inductance parameters of transmission lines based on GPS technology. IEEE Transactions on Power Delivery. Vol. 2 3(2008) No. 3, p.1288-1295.

[14]HU Zhijian, LIU Meiguan, ZHANG Chengxue, et al. Principles and realization of live line measurement to parameters of transmission lines with mutual inductance. Vol. 23 (1999) No. 24, p.32-35. 